\title{
Capítulo 11
}

Monitoramento de ecossistemas bentônicos estuarinos

\author{
Angelo F. Bernardino \\ Francisco Barros \\ Larisse F. Perez \\ Sergio A. Netto \\ Leonir A. Colling \\ Paulo R. Pagliosa \\ Paulo C. Lana \\ Rafaela C. Maia \\ Ronaldo A. Christofoletti \\ José Souto R. Filho \\ Tânia M. Costa
}

\section{SciELO Books / SciELO Livros / SciELO Libros}

BERNARDINO, AF., et al. Monitoramento de ecossistemas bentônicos estuarinos. In: TURRA, A., and DENADAI, MR., orgs. Protocolos para o monitoramento de habitats bentônicos costeiros - Rede de Monitoramento de Habitat Bentônicos Costeiros - ReBentos [online]. São Paulo: Instituto Oceanográfico da Universidade de São Paulo, 2015, pp. 134-154. ISBN 978-85-98729-25-1. Available from SciELO Books <http://books.scielo.org $>$.

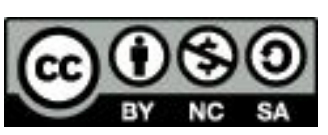

All the contents of this work, except where otherwise noted, is licensed under a Creative Commons Attribution-Non Commercial-ShareAlike 3.0 Unported.

Todo o conteúdo deste trabalho, exceto quando houver ressalva, é publicado sob a licença Creative Commons Atribuição Uso Não Comercial - Partilha nos Mesmos Termos 3.0 Não adaptada.

Todo el contenido de esta obra, excepto donde se indique lo contrario, está bajo licencia de la licencia Creative Commons Reconocimento-NoComercial-CompartirIgual 3.0 Unported. 


\section{SciELO Books / SciELO Livros / SciELO Libros}

BERNARDINO, AF., et al. Monitoramento de ecossistemas bentônicos estuarinos. In: TURRA, A., and DENADAI, MR., orgs. Protocolos para o monitoramento de habitats bentônicos costeiros - Rede de Monitoramento de Habitat Bentônicos Costeiros - ReBentos [online]. São Paulo: Instituto Oceanográfico da Universidade de São Paulo, 2015, pp. 134-154. ISBN 978-85-98729-25-1. Available from SciELO Books <http://books.scielo.org>.

\section{(2)(1)(2)}

All the contents of this work, except where otherwise noted, is licensed under a Creative Commons Attribution-Non Commercial-ShareAlike 3.0 Unported.

Todo o conteúdo deste trabalho, exceto quando houver ressalva, é publicado sob a licença Creative Commons Atribuição Uso Não Comercial - Partilha nos Mesmos Termos 3.0 Não adaptada.

Todo el contenido de esta obra, excepto donde se indique lo contrario, está bajo licencia de la licencia Creative Commons Reconocimento-NoComercial-CompartirIgual 3.0 Unported. 


\section{CAPÍTULO 11}

\section{MONITORAMENTO DE ECOSSISTEMAS BENTÔNICOS ESTUARINOS}

Angelo F. Bernardino, Francisco Barros, Larisse F. Perez, Sergio A. Netto, Leonir A. Colling, Paulo R. Pagliosa, Paulo C. Lana, Rafaela C. Maia, Ronaldo A. Christofoletti, José Souto R. Filho, Tânia M. Costa

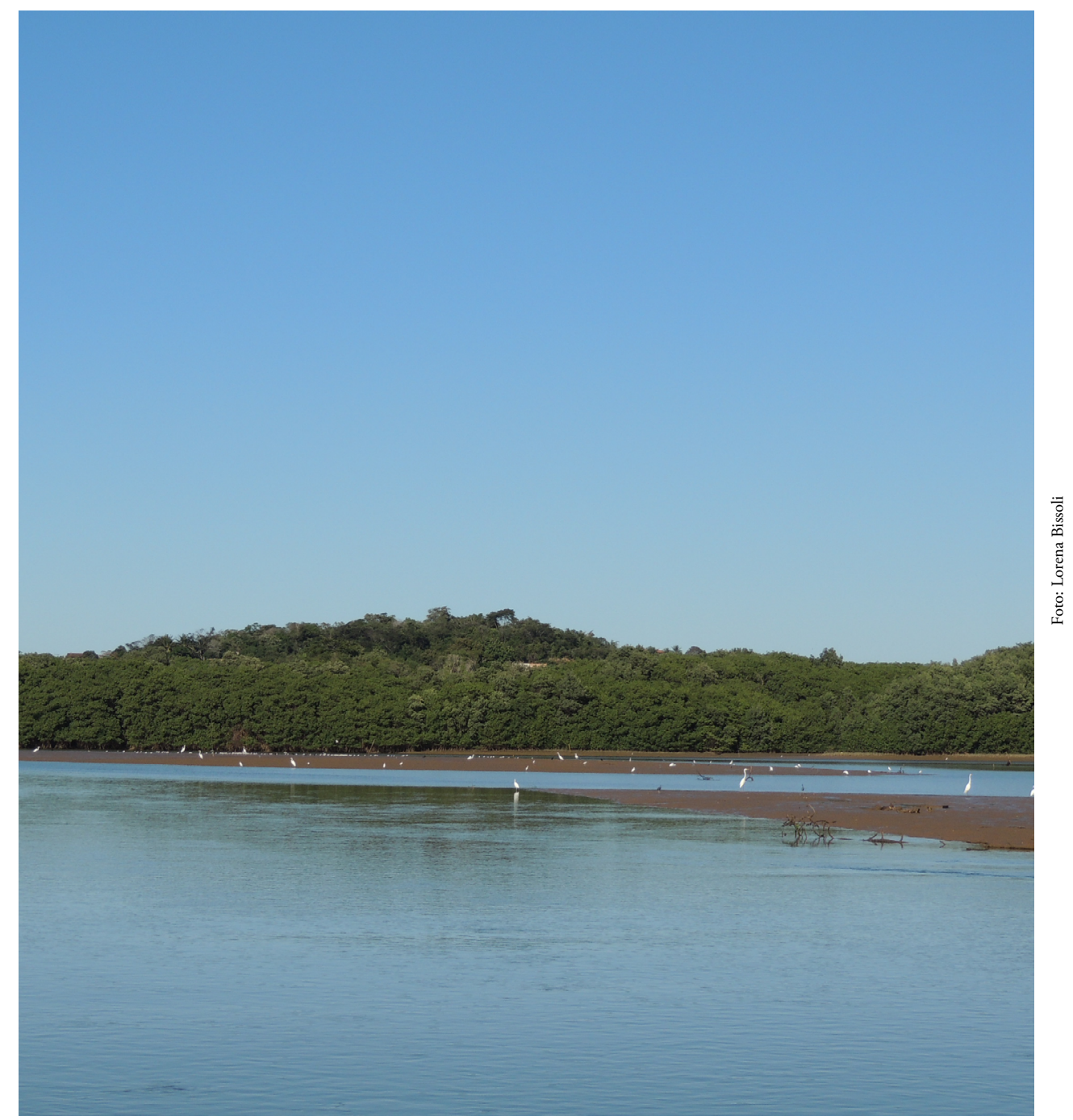

\section{INTRODUÇÃO}

Estuários são ecossistemas de transição continente-oceano com alta produtividade biológica, distinta biodiversidade bentônica e que sustentam importantes bens e serviços ambientais à humanidade. Estuários estão intimamente associados à dinâmica físico-química costeira, incluindo interaçóes entre variáveis biológicas, meteorológicas e oceanográficas. Esses processos interagem e estruturam as comunidades bentônicas estuarinas através de complexas interaçóes em diferentes escalas espaciais (e.g. local, regional e global) e temporais (e.g. diária, sazonal e anual). 
Nas últimas décadas, atividades antrópicas têm contribuído para a transformação dos ecossistemas estuarinos, seja pela massiva introdução de efluentes, ocupação urbana e remoção de áreas vegetadas, intervençóes na hidrodinâmica costeira, ou por crescentes atividades pesqueiras e de maricultura. Além dos intensos impactos locais e regionais sobre os estuários, mudanças climáticas globais também poderiam exercer pressão sobre a dinâmica desses ecossistemas e suas comunidades de uma maneira, escala e intensidades ainda pouco estudadas. Alteraçóes climáticas como mudanças na temperatura, no nível médio do mar e no regime pluviométrico, têm potencial para alterar os regimes hidrológicos e biogeoquímicos existentes, representando talvez a maior ameaça aos ecossistemas estuarinos em escala global (Nicholls et al., 1999; Gilman et al., 2008). Mudanças na hidrologia estuarina podem, por exemplo, alterar a salinidade média estuarina, modificando padrôes de distribuição e produtividade de invertebrados e alterar a cadeia trófica estuarina. Os impactos resultantes de alteraçôes climáticas são de difícil previsão e poderão ser mais acentuados em estuários já impactados regionalmente, colocando seriamente em risco a biodiversidade bentônica estuarina e seu equilíbrio ecológico.

O planejamento para estudos de mudanças climáticas em ecossistemas bentônicos estuarinos requer prévio entendimento dos principais agentes que regulam a dinâmica estuarina, além das possíveis mudanças regionais esperadas entre esses agentes. O Grupo de Trabalho (GT) Estuários identificou as principais vulnerabilidades de ecossistemas bentônicos estuarinos frente às mudanças no clima e elencou duas variáveis centrais na dinâmica estuarina a serem monitoradas: temperatura atmosférica e salinidade. A priorização desses parâmetros justifica-se pela sua relativa facilidade de monitoramento local e regional, assim como pela disponibilidade de dados históricos através de redes meteorológicas nacionais (Bernardino, et al., 2015; no prelo)

Considerando a ampla heterogeneidade climática existente no Brasil, o GT utilizou referências atuais (Marengo et al., 2010; McGlone \& Vuille, 2012), que melhor apresentam cenários de alteraçóes climáticas atmosféricas e sua relação com fenômenos de ENSO (El Niño Southern Oscilation) previstos para o Brasil entre os anos de 2071 a 2100, na hipótese de manutenção do cenário de altas emissôes de $\mathrm{CO}_{2}$ feitas pelo IPCC (cenário A2, IPCC, 2007). Além da evidente importância dos parâmetros climáticos de temperatura e pluviometria para o funcionamento de ecossistemas bentônicos estuarinos, o GT identificou a elevação no nível médio do mar como um terceiro importante componente, e que também possui forte suporte científico (e.g. Meier et al., 2007; IPCC, 2007). Porém, quantificar e monitorar alteraçóes de nível médio do mar em regiôes costeiras é complexo e necessita de rigoroso acompanhamento técnico e de equipamentos de custo elevado. Assim, visando a ampla execução deste protocolo, não incluímos o monitoramento do nível médio do mar nos monitoramentos estuarinos. Em algumas previsóes climáticas é frequente observarmos tendências heterogêneas (i.e. na intensidade de mudança) ou até opostas regionalmente. Por exemplo, modelos climáticos indicam diminuição da pluviosidade média ao longo de grande parte do Norte, Nordeste e Sudeste do Brasil, mas com tendência oposta na regiáo Sul (Marengo et al., 2010 - Tabela 11-1). No entanto, as previsóes de aumento de temperatura média e elevação do nível do mar são consistentes em todo o território nacional e suas regióes costeiras (Angulo \& Lessa, 1997; Meier et al., 2007; Raicich, 2008; Marengo et al., 2010).

Tabela 11-1. Previsóes climáticas baseadas em modelos regionais e globais para as regióes brasileiras

\begin{tabular}{|l|c|c|c|c|}
\hline & Norte & Nordeste & Sudeste & Sul \\
\hline Pluviosidade & - & - & - & + \\
\hline Temperatura atmosférica & + & + & + & + \\
\hline $\begin{array}{l}\text { Variação do nível médio do } \\
\text { mar }\end{array}$ & + & + & + & + \\
\hline $\begin{array}{l}\text { Previsão de efeitos no ecos- } \\
\text { sistema bêntico estuarino }\end{array}$ & $\begin{array}{l}\text { Alterações na salinidade média; mudanças no metabolismo } \\
\text { e produtividade de espécies bentônicas, deslocamento de } \\
\text { populações entre regiões salinas, invasão de espécies. }\end{array}$ \\
\hline
\end{tabular}


A partir das projeçôes climáticas disponíveis, o GT procurou por modelos biológicos (i.e. no nível de indivíduos, populaçôes ou comunidades) com íntima relação com as variáveis de interesse (temperatura e salinidade). Esses modelos foram exaustivamente debatidos, no entanto, o grupo entende que a definição dos melhores modelos para estudos de longo prazo necessitam de um protocolo de amostragem simplificado e passível de ser praticado por todos os pesquisadores de estuários. Dessa maneira, o protocolo apresentado a seguir satisfaz os objetivos da ReBentos apresentando recomendaçóes mínimas de amostragem que podem ser facilmente replicadas por pesquisadores em todo o Brasil.

Este protocolo apresenta de forma objetiva as etapas para utilizar animais bentônicos estuarinos como modelos para acompanhamento de mudanças na pluviosidade e temperatura (além de outras co-variáveis) em estuários brasileiros. Trata-se de um protocolo de simples execução, justificável cientificamente e ao mesmo tempo atrativo a novos participantes, amplificando o alcance da ReBentos. O objetivo deste protocolo é guiar o pesquisador, em qualquer regiáo costeira brasileira, para iniciar o monitoramento de possíveis efeitos produzidos por mudanças climáticas em ecossistemas estuarinos brasileiros, utilizando animais bentônicos como indicadores. Como detalhado acima, as hipóteses de estudo que suportam as variáveis de interesse da ReBentos GT-Estuários são baseadas nas previsóes climáticas a partir de modelos regionais e globais (Marengo et al., 2010; McGlone \& Vuille, 2012) e podem ser, simplificadamente, organizadas por região do território nacional (Tabela 11-1).

Baseando-se nessas previsóes, pesquisadores de todas as regióes do Brasil podem escolher um estuário para estudo e iniciar o monitoramento. O número de indicadores a serem monitorados em cada estuário pode ser determinado por cada grupo de pesquisa, considerando a logística, infraestrutura e os recursos humanos disponíveis. Porém, para a escolha de estuários e definições de sítios de estudo, fazemos uma série de recomendaçóes, conforme a seguir.

\section{MÉTODOS}

\section{Planejamento Amostral}

Em um cenário ideal, o GT Estuários deve contar com monitoramentos em todas as regiôes da costa brasileira (i.e. N, NE, SE e S) e, em cada região, a escolha de estuários para monitoramento deve primeiramente satisfazer: 1) estuários com mínima influência antrópica (e.g. urbanização, poluição), e 2) priorizar estuários inseridos em áreas de preservação estadual ou federal, quando possível. No segundo caso, além da necessidade de autorização dos órgãos ambientais competentes (e.g. ICMBio, IEMAs), a coordenação da ReBentos, quando solicitada, poderá reforçar o contato com os órgáos públicos e solicitar apoio logístico local, caso existente e disponível.

Em cada estuário será definida uma "área”, fixa ao longo do tempo, para monitoramento. Essa área de estudo deverá estar localizada no setor euhalino ou baixo estuário (sensu McLusky \& Eliott, 2006). Essa padronização evita a introdução de fatores de confusão decorrentes da ampla variabilidade físico-química estuarina e permite significativa redução no esforço amostral do estudo. É desejável que essa área contenha uma franja de 250-350 metros de planície de maré seguida por manguezal ou marisma, sendo representativa dos ecossistemas dominantes (ou paisagem) no estuário em questão.

Uma vez definida a localização da área de estudo, ela será dividida em três "sítios" (Figura 11-1). Cada sítio de estudo deve conter um trecho de franja de aproximadamente 50 metros lineares, contíguos e paralelos ao estuário. Cada sítio deverá também ter uma extensão de 50 metros perpendicular ao estuário, ou seja, em direção ao bosque de mangue ou marisma. Assim, cada área irá conter 3 sítios de estudo, espaçados entre si por 50-100 metros de distância. Cada sítio irá conter, perpendicularmente ao canal estuarino, habitats bentônicos não-vegetados e vegetados (Figura 11-1). Esses habitats, então, ilustram basicamente ecossistemas distintos presentes em um único sítio de estudo, e serão amostrados de maneira distinta como exemplificado a seguir. 
De forma resumida, alguns princípios práticos para determinação da área e dos sítios de estudo incluem:

- No baixo estuário uma área será estabelecida e 3 sítios serão delimitados por uma região contígua de $50 \mathrm{~m}$ x $50 \mathrm{~m}$ (largura x comprimento);

- Cada sítio abrigará 2 habitats (vegetado e não-vegetado). A escolha do tipo de vegetação depende de sua dominância no local;

- Cada sítio amostral (mínimo n=3) deve possuir uma distância de 50-100 metros entre si (Figura 11-1). Em marismas com reduzida extensão, os sítios amostrais deverão ocorrer em um mesmo banco (ANEXO 11-II);

- Na região de borda dentro das áreas vegetadas de manguezal os primeiros 10 metros devem ser desconsiderados (zona de borda na Figura 11-1). Ressalta-se que na maioria dos casos essa exclusão é desnecessária quando a área vegetada for uma marisma;

- Os sítios de estudo podem ser diferenciados em seu desenho espacial, descrito para permitir a amostragem de gastrópodes (Littoraria) e caranguejos $\left(U_{c a}\right)$ em locais onde estas espécies não ocorrem contiguamente, respeitando-se a amostragem no setor euhalino estuarino. Por exemplo, em sítios onde $U c a$ spp. não ocorre em baixios lamosos, pode se realizar a amostragem em áreas de transição entre bosques e restinga.

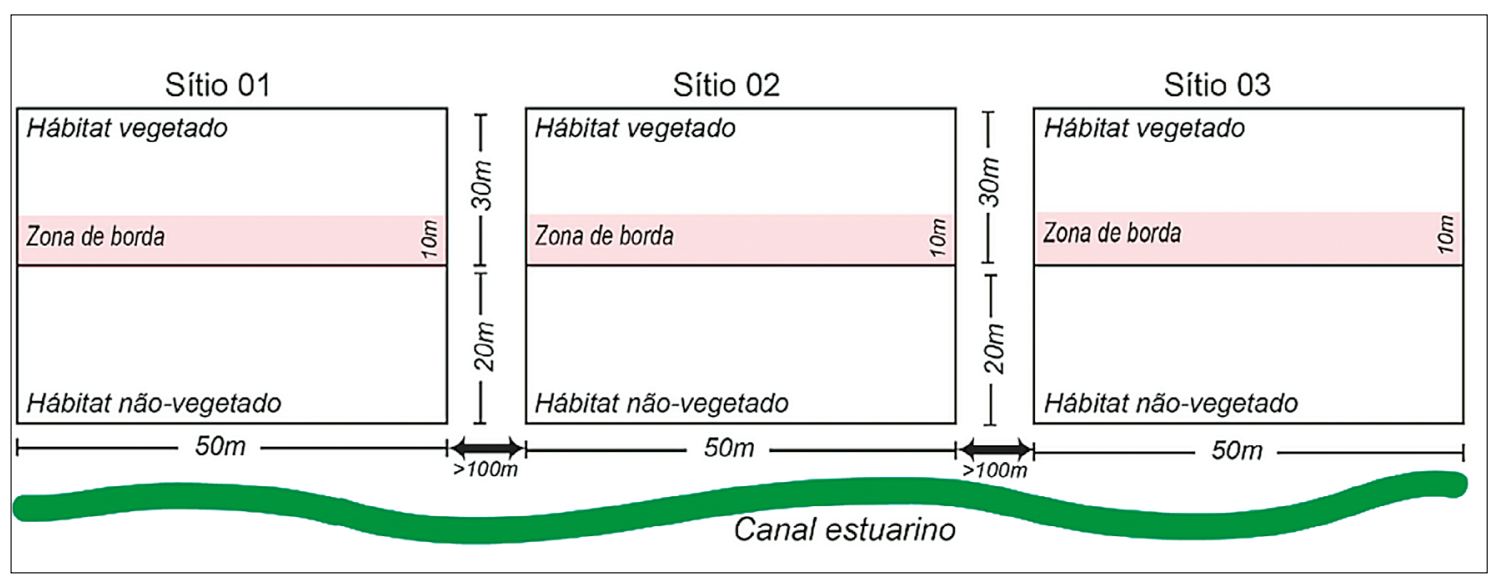

Figura 11-1. Esquema de organização espacial dos sítios de amostragem no baixo estuário.

\section{Amostragem}

Cada sítio de estudo irá incorporar substratos caracterizados por fundos vegetados e não-vegetados, sejam estes contíguos (Figura 11-1) ou separados. Os habitats vegetados serão representados por manguezais (limitadas ao sul até o estado de Santa Catarina; Schaeffer-Novelli et al., 1990), e por marismas (Laguna, em Santa Catarina até o estado do Rio Grande do Sul). Os métodos de amostragem e as populaçóes-alvo diferem de acordo com o tipo de cobertura vegetal (i.e. habitats) como descrito no ANEXO 11-II. Os habitats não-vegetados são representados tipicamente pelas planícies de maré localizadas nas margens dos canais estuarinos.

A fauna bentônica alvo desse monitoramento incluirá animais da megafauna comumente presentes em árvores de mangue, como gastrópodes Littoraria angulifera, ou observados em sedimentos náo vegetados, como caranguejos do gênero Uca spp. (existem cerca de dez espécies descritas no Brasil; Melo, 1996; Thurman, et al., 2013). Em algumas localidades onde não ocorre Uca nas margens dos canais estuarinos, os habitats não vegetados podem ser representados por apicuns ou regiôes de transição entre manguezal e restinga que abriguem grandes densidades desses caranguejos. Nesses casos, deve-se tomar o cuidado para estabelecer os sítios amostrais em habitats similares dentro de uma mesma área de estudo 
(e.g. três sítios em região de transição manguezal-restinga dentro da Baía de Paranaguá). Da mesma maneira, a amostragem de animais da epifauna arborícola (gastrópodes) ou sedimentar ( $U_{c a}$ ) não necessita ocorrer em um mesmo sítio em locais onde estes animais não co-ocorrem, mas deve-se observar que todos os sítios amostrais devem se localizar dentro do baixo estuário.

A amostragem da fauna é específica para cada habitat:

\section{Habitats não Vegetados}

Nos sedimentos não-vegetados, serão amostradas caranguejos do gênero $U c a$ e a macroinfauna $(>500 \mu \mathrm{m})$. Em cada sítio, a amostragem de $U c a$ será realizada pela contagem de indivíduos em quadrats aleatórios $(75 \times 75 \mathrm{~cm})$. Após o posicionamento aleatório do quadrat, prosseguir com contagem das tocas e a captura dos indivíduos, tomando cuidado para diferenciar eventuais aberturas no sedimento criadas por animais depositívoros como bivalves e anelídeos (estes buracos náo devem ser contados como tocas na medida do possível). A captura dos ucas será manual e os animais coletados podem ser acondicionados em sacos plásticos e congelados em laboratório até análise. Recomenda-se colocar folhas ou detritos nos sacos para evitar perda de partes do animal. Durante o campo, os animais podem ser colocados em um isopor com gelo logo após coleta para baixar o ritmo de atividade.

A macroinfauna também será amostrada nos habitats não-vegetados e nas marismas (ANEXO 11-II) objetivando acompanhar densidade, biomassa e produtividade secundária dos animais. Em cada sítio, usar um tubo (PVC ou acrílico) de $10 \mathrm{~cm}$ de diâmetro e introduzi-lo a $10 \mathrm{~cm}$ de profundidade no sedimento. Serão coletadas três amostras de macroinfauna por sítio, de forma aleatória, em locais sem perturbação por pisoteio. Essas amostras podem ser acondicionadas em sacos plásticos, e fixadas em formol diluído em água do mar a 4\%. Após fixação (> 48 horas), as amostras podem ser peneiradas com água corrente em malha de $500 \mu \mathrm{m}$, e preservadas em álcool $70 \%$ até análise.

\section{Habitats Vegetados}

Em bosques de mangue serão amostrados gastrópodes arborícolas do gênero Littoraria. Em cada sítio com presença de mangue, ao longo de um transecto de $20 \mathrm{~m}$, perpendicular ao estuário, os gastrópodes aderidos às raízes e aos troncos das árvores, até o nível da altura do peito (aproximadamente 1-1,5 m acima do solo), deveráo ser amostrados. Aproximadamente 20 indivíduos serão amostrados por transecto, totalizando aproximadamente 60 indivíduos coletados na área de estudo.

Nas marismas, a amostragem da macroinfauna consistirá de três amostras aleatórias tomadas com tubo (PVC ou acrílico) de $10 \mathrm{~cm}$ de diâmetro a $10 \mathrm{~cm}$ de profundidade no banco. A fixação e preservação das amostras são idênticas às descritas acima.

\section{Repetição da Amostragem no Tempo}

O objetivo da ReBentos é monitorar variaçóes decorrentes do clima, ou seja, em escalas de décadas. Assim, para viabilizar o monitoramento de longo prazo, o esforço amostral mínimo em cada área de estudo deverá incluir duas campanhas de coleta por período, sempre realizadas ao final de temporadas anuais de chuva e seca. De acordo com a identificação histórica dessas temporadas durante o ano em cada regiáo de estudo, as estaçóes chuvosas e secas estão concentradas, de acordo com a regiáo, nos meses (Tabela 11-2): 
Tabela 11-2. Períodos com dominância de chuvas e secas nas regiôes brasileiras

\begin{tabular}{|l|c|c|}
\hline Região & Chuva & Seca \\
\hline Norte (incluindo MA, PI e CE) & Fev-Mar & Out-Nov \\
\hline Nordeste (RN a BA) & Jul-Ago & Jan-Mar \\
\hline Sudeste & Fev-Mar & Set-Out \\
\hline Sul & Jul-Ago & Fev-Mar \\
\hline
\end{tabular}

Dessa maneira teríamos, por exemplo, em um estuário localizado na região Sudeste, duas campanhas de coleta nos meses de fevereiro e março, representando o período chuvoso; e outras duas campanhas de coleta nos meses de setembro e outubro, representando o período seco.

\section{ANÁLISES LABORATORIAIS E DE DADOS}

\section{Epifauna}

Nas amostras de epifauna (Uca e Littoraria), a coleta e análise de dados deverá incluir: (1) número de indivíduos por espécies ou morfotipo (riqueza); (2) tamanho (comprimento e largura - C x L) e biomassa (peso seco ou peso seco livre de cinzas) e (3) cálculo de produção secundária a partir da biomassa (ANEXO 11-III).

A epifauna deve ser identificada e ter o sexo determinado (caranguejos), medida (comprimento e largura - Figura 11-2), e pesada sob balança com precisão mínima de 0,1 mg. Para pesagem úmida, estabelecer um tempo de 2 min para estabilização do peso e anotação do valor.

A

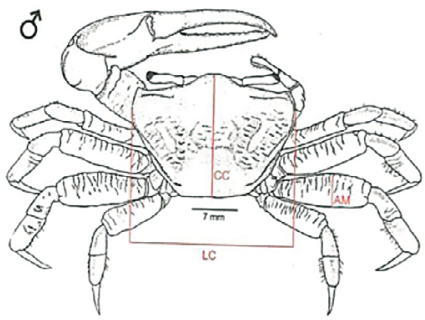

B \&

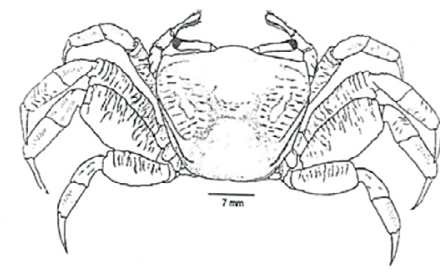

C

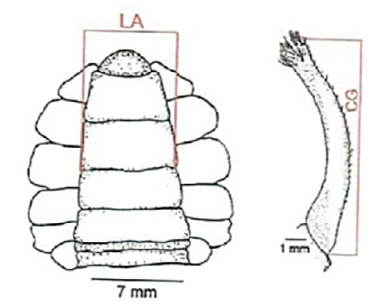

D

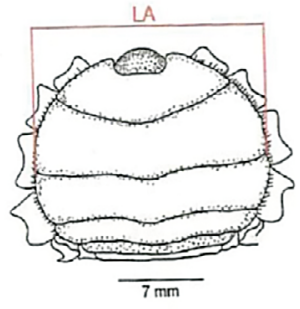

E

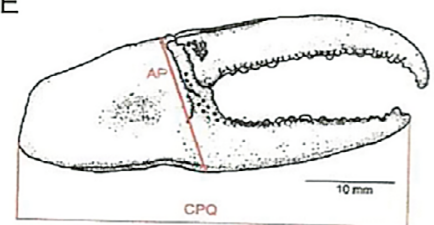

$\mathrm{F}$

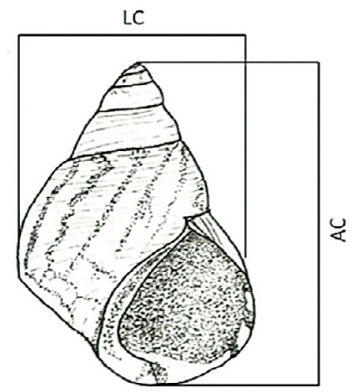

Figura 11-2. Guia visual de mediçôes a serem retiradas de Caranguejos Uca spp. (painéis A-E) e de Littoraria angulifera (painel F). A. Vista dorsal de um macho, LC = largura da carapaça, $C C=$ comprimento da carapaça, $A M=$ altura do mero do 3o pereópodo; B. Vista dorsal de uma fêmea; C. Abdome de um macho, LA= largura do abdome na base do 50 somito abdominal, $\mathrm{CG}=$ comprimento do gonóporo; $\mathrm{D}$. Abdome de uma fêmea, $\mathrm{LA}=$ largura do abdome na base do 5 o somito abdominal; $\mathrm{E}$. Quelípodo maior do macho, $\mathrm{AP}=$ altura do própodo, $\mathrm{CPQ}=$ comprimento do própodo quelar; $\mathrm{F}$. Concha de Littoraria angulifera, $\mathrm{LC}=$ largura da concha, $\mathrm{AC}=$ altura da concha. Imagens: T. M. Costa e R. C. Maia. 


\section{Macroinfauna}

As amostras da macroinfauna fixadas em formalina $4 \%$ devem ser lavadas na malha de $0,5 \mathrm{~mm}$ e preservadas em álcool $70 \%$. Os animais devem ser triados e identificados e suas biomassas determinadas em grupos taxonômicos mais inclusivos (classe ou ordem; ver "Referências Bibliográficas para Taxonomia”). Os dados de abundância e biomassa deverão ser lançados em planilhas disponibilizadas (Tabela 11-5 - ver adiante), e serão utilizados posteriormente para cálculo de produção secundária. Os protocolos de cálculo de produção secundária baseiam-se nas medidas de tamanho e/ou peso dos animais (ANEXO 11-III, relações produção x biomassa - P/B; Brey, 2001).

\section{Sedimentos}

Para caracterizar as áreas de estudo, amostras compostas de sedimentos podem ser coletadas uma vez ao ano. Uma amostra composta para análises sedimentares consiste em misturar um tubo de sedimento (10 cm de diâmetro x $10 \mathrm{~cm}$ de profundidade) amostrado em cada um dos 3 sítios de uma área, misturados, e retirada uma quantidade aproximada de 100 gramas. Essa amostra composta deve ser levada para laboratório e congelada até a realização de análises granulométricas e de matéria orgânica. As análises sedimentares para determinação de granulometria e conteúdo de matéria orgânica $(\mathrm{MO})$ deverão obedecer ao protocolo de determinação das propriedades sedimentológicas (ANEXO 11-IV). As amostras serão analisadas por peneiramento (fração areia) e pipetagem ou difração a laser (fração lama) e conteúdo de MO.

\section{CARActerização e Monitoramento Físico-Químico-Geológico}

Reconhecendo a ampla dinâmica físico-química e geológica dos estuários a serem monitorados, recomenda-se adicionalmente um protocolo ambiental mínimo para cada área de estudo, que deverá ser realizado apenas na primeira campanha visando obter dados descritivos das áreas. Na primeira visita e no momento de escolha dos sítios, os pesquisadores deverão identificar a estrutura de bosque de mangue ou marismas, medindo variáveis que indiquem a composição, densidade e altura da vegetação (Mangue ou Spartina; ANEXO 11-I).

Os pesquisadores da rede devem auxiliar na consolidação e obtenção de dados físico-químicos constantes em suas áreas de estudo. Dessa forma, é recomendado que cada pesquisador obtenha periodicamente os seguintes parâmetros em seus estuários:

- Dados de temperatura, altura e períodos de ondas, incidência de ventos e pluviosidade deverão ser obtidos, continuamente, em serviços de previsóes do tempo e estudos climáticos*. Como esses dados ficam disponíveis eletronicamente por um período de 90 dias desde sua aquisição, cabe aos pesquisadores realizarem sistemática aquisiçáo desses dados ao longo dos anos (em torno de quatro vezes ao ano);

- Quantificar o peso úmido e o peso seco do biodetrito sedimentar proveniente das amostras de macrofauna coletadas por tubos, retirando o detrito retido nas malhas após peneiramento da fauna;

- Dados de vazão do estuário, que podem ser adquiridos junto à Agência Nacional das Águas (ANA - www.ana.gov.br).

Esses dados poderão ser arquivados e disponibilizados no Banco de Dados da ReBentos para a realização de sínteses e preparação de artigos, sendo garantida a autoria a quem gerou os dados (ver Capítulo 1). Obviamente cada pesquisador tem a liberdade de utilizá-los em trabalhos correlatos em suas áreas de estudo.

* CPTEC http://www.cptec.inpe.br

SIMCos http://www.lamma.ufrj.br/sites/simcos/paginas/sistema/index.html Instituto Nacional de Meteorologia http://www.inmet.gov.br/html/rede_obs.php

Climatempo http://www.climatempo.com.br/ 


\section{SistematizaçÃo na AnotaÇão de Dados e demais AnÁlises}

Após a coleta, análise e obtenção dos dados, é fundamental a sistematização de tabelas para tornar compatíveis as comparaçôes regionais e temporais entre equipes do GT Estuários. Para facilitar a anotação de dados, são disponibilizadas aqui planilhas modelo para o preenchimento dos dados:

- Tabela 11-3: planilha de registro de amostragem;

- Tabela 11-4: planilha de dados de Uca;

- Tabela 11-5: planilha de dados de macrofauna;

- Tabela 11-6: planilha de dados de Littoraria angulifera.

Após a aquisição de dados, a rede viabilizará a análise conjunta de dados entre seus membros e todos os pesquisadores com dados disponíveis serão convidados a participar dos produtos gerados. A ReBentos irá disponibilizar formas de armazenamento e depositório de dados online (ver seção "Banco de Dados” - Capítulo 1). 
Tabela 11-3. Planilha para controle das atividades de laboratório e análise de dados

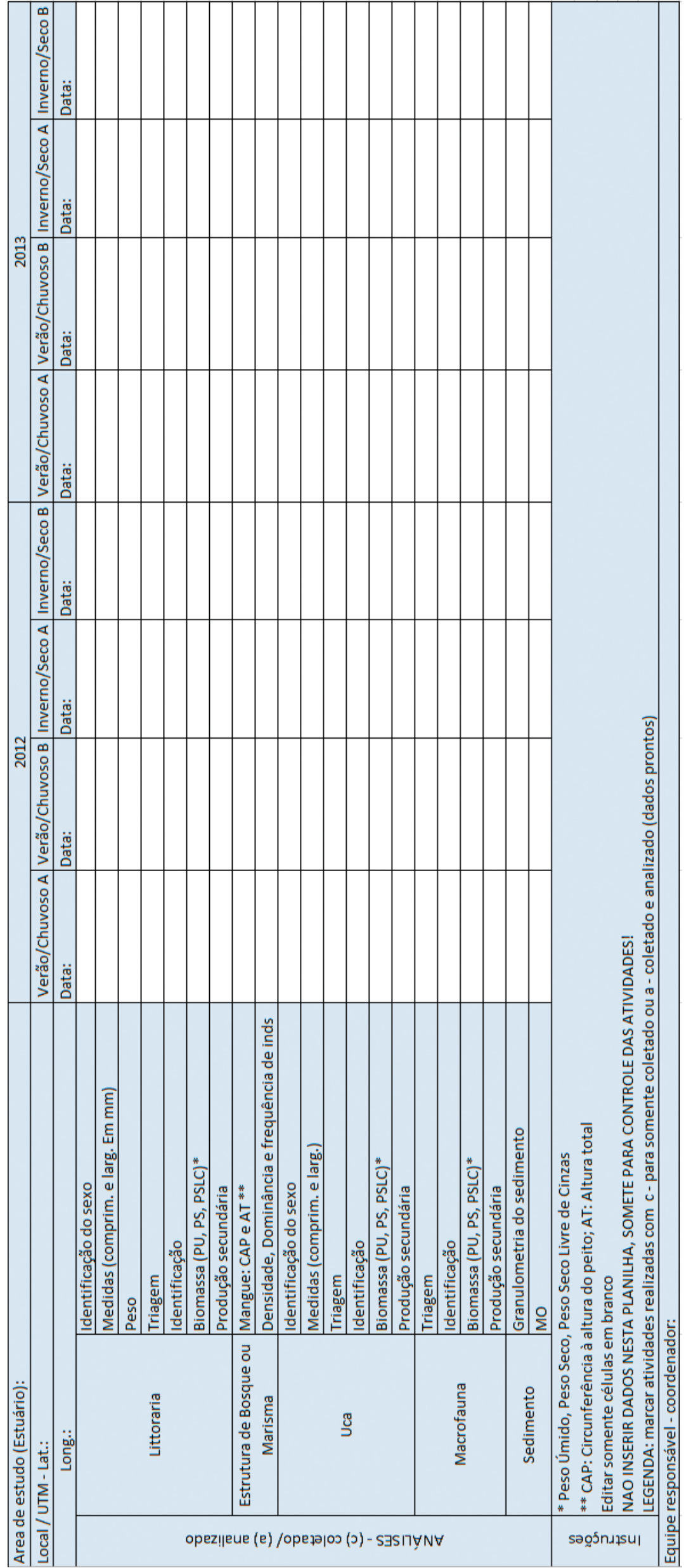


Tabela 11-4. Planilha para inserção dos dados sobre o caranguejo Uca

\begin{tabular}{|c|c|c|c|c|c|c|c|c|c|c|c|c|}
\hline & & & & & Uca & & & & & & & \\
\hline Estação: & & & & & & & & & & & & \\
\hline Ano: & & & & & & & & & & & & \\
\hline Campanha: & & & & & & & & & & & & \\
\hline & & & & & Sítio 1 & & & & & & & \\
\hline Espécies encontra & & & & & & & & & & & & \\
\hline Amostra & Indivíduo & LC & CC & $\mathrm{AM}$ & LA (M) & CG & LA (F) & AP & $\mathrm{CPQ}$ & PU & PS & PSLC \\
\hline Quadrat 1 & & & & & & & & & & & & \\
\hline ( $\mathrm{n}^{\circ}$ de tocas) & & & & & & & & & & & & \\
\hline & & & & & & & & & & & & \\
\hline & & & & & & & & & & & & \\
\hline & & & & & & & & & & & & \\
\hline & & & & & & & & & & & & \\
\hline & & & & & & & & & & & & \\
\hline & & & & & & & & & & & & \\
\hline & & & & & & & & & & & & \\
\hline Quadrat 2 & & & & & & & & & & & & \\
\hline ( $\mathrm{n}^{\circ}$ de tocas) & & & & & & & & & & & & \\
\hline & & & & & & & & & & & & \\
\hline & & & & & & & & & & & & \\
\hline & & & & & & & & & & & & \\
\hline & & & & & & & & & & & & \\
\hline & & & & & & & & & & & & \\
\hline & & & & & & & & & & & & \\
\hline & & & & & & & & & & & & \\
\hline Quadrat 3 & & & & & & & & & & & & \\
\hline ( $n^{\circ}$ de tocas) & & & & & & & & & & & & \\
\hline & & & & & & & & & & & & \\
\hline & & & & & & & & & & & & \\
\hline & & & & & & & & & & & & \\
\hline & & & & & & & & & & & & \\
\hline & & & & & & & & & & & & \\
\hline & & & & & & & & & & & & \\
\hline & & & & & Sítio 2 & & & & & & & \\
\hline Espécies encontra & & & & & & & & & & & & \\
\hline Amostra & Indivíduo & LC & CC & $\mathrm{AM}$ & LA (M) & CG & LA (F) & $\mathrm{AP}$ & $\mathrm{CPQ}$ & PU & PS & PSLC \\
\hline Quadrat 1 & & & & & & & & & & & & \\
\hline ( $n^{\circ}$ de tocas) & & & & & & & & & & & & \\
\hline & & & & & & & & & & & & \\
\hline & & & & & & & & & & & & \\
\hline & & & & & & & & & & & & \\
\hline & & & & & & & & & & & & \\
\hline & & & & & & & & & & & & \\
\hline & & & & & & & & & & & & \\
\hline & & & & & & & & & & & & \\
\hline Quadrat 2 & & & & & & & & & & & & \\
\hline ( $\mathrm{n}^{\circ}$ de tocas) & & & & & & & & & & & & \\
\hline & & & & & & & & & & & & \\
\hline & & & & & & & & & & & & \\
\hline & & & & & & & & & & & & \\
\hline & & & & & & & & & & & & \\
\hline & & & & & & & & & & & & \\
\hline & & & & & & & & & & & & \\
\hline & & & & & & & & & & & & \\
\hline Quadrat 3 & & & & & & & & & & & & \\
\hline ( $n^{\circ}$ de tocas) & & & & & & & & & & & & \\
\hline & & & & & & & & & & & & \\
\hline & & & & & & & & & & & & \\
\hline & & & & & & & & & & & & \\
\hline & & & & & & & & & & & & \\
\hline & & & & & & & & & & & & \\
\hline & & & & & & & & & & 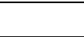 & & \\
\hline $\begin{array}{l}\text { Legenda: LC - larg } \\
\text { LA (F) - largura ab } \\
\text { cinzas. }\end{array}$ & $\begin{array}{l}\text { apaça; CC } \\
\text { fêmea; A }\end{array}$ & com & nto & $\begin{array}{l}\text { a; } \mathrm{AN} \\
-\mathrm{con}\end{array}$ & $\begin{array}{l}\text { altura } \\
\text { riment }\end{array}$ & ; LA & M)- larg & dom & acho; & $\begin{array}{l}\text { compr } \\
\text { seco; I }\end{array}$ & to & $\begin{array}{l}\text { podo; } \\
\text { seco livre }\end{array}$ \\
\hline
\end{tabular}


Tabela 11-5. Planilha para inserção dos dados de biomassa da macroinfauna.

\begin{tabular}{|c|c|c|c|c|c|}
\hline \multicolumn{6}{|c|}{ Macrofauna } \\
\hline \multicolumn{6}{|l|}{ Estação: } \\
\hline \multicolumn{6}{|l|}{ Ano: } \\
\hline \multicolumn{6}{|l|}{ Campanha: } \\
\hline \multicolumn{6}{|c|}{ Sítio 1} \\
\hline \multirow{5}{*}{ Corer A } & & Abundância & $\mathrm{PU}$ & PS & PSLC \\
\hline & Aciculata & & & & \\
\hline & Scolecidae & & & & \\
\hline & Canalipalpata & & & & \\
\hline & Fragmentos & & & & \\
\hline & & & & & \\
\hline \multirow{4}{*}{ Corer B } & Aciculata & & & & \\
\hline & Scolecidae & & & & \\
\hline & Canalipalpata & & & & \\
\hline & Fragmentos & & & & \\
\hline & & & & & \\
\hline \multirow{4}{*}{ Corer C } & Aciculata & & & & \\
\hline & Scolecidae & & & & \\
\hline & Canalipalpata & & & & \\
\hline & Fragmentos & & & & \\
\hline \multicolumn{6}{|c|}{ Sítio 2} \\
\hline \multirow{5}{*}{ Corer A } & & Abundância & PU & PS & PSLC \\
\hline & Aciculata & & & & \\
\hline & Scolecidae & & & & \\
\hline & Canalipalpata & & & & \\
\hline & Fragmentos & & & & \\
\hline \multirow{4}{*}{ Corer B } & Aciculata & & & & \\
\hline & Scolecidae & & & & \\
\hline & \begin{tabular}{|l|} 
Canalipalpata \\
\end{tabular} & & & & \\
\hline & Fragmentos & & & & \\
\hline \multirow{5}{*}{ Corer C } & & & & & \\
\hline & Aciculata & & & & \\
\hline & Scolecidae & & & & \\
\hline & Canalipalpata & & & & \\
\hline & Fragmentos & & & & \\
\hline \multicolumn{6}{|c|}{ Sítio 3} \\
\hline \multirow{5}{*}{ Corer A } & & Abundância & $\mathrm{PU}$ & PS & PSLC \\
\hline & Aciculata & & & & \\
\hline & Scolecidae & & & & \\
\hline & Canalipalpata & & & & \\
\hline & Fragmentos & & & & \\
\hline \multirow{5}{*}{ Corer B } & & & & & \\
\hline & Aciculata & & & & \\
\hline & Scolecidae & & & & \\
\hline & Canalipalpata & & & & \\
\hline & Fragmentos & & & & \\
\hline \multirow{5}{*}{ Corer C } & & & & & \\
\hline & Aciculata & & & & \\
\hline & Scolecidae & & & & \\
\hline & Canalipalpata & & & & \\
\hline & Fragmentos & & & & \\
\hline \multicolumn{6}{|c|}{$\begin{array}{l}\text { Legenda: } \\
\text { PU = peso úmido; } \\
\text { PS = peso seco; } \\
\text { PSLC = peso seco livre de cinzas }\end{array}$} \\
\hline
\end{tabular}


Tabela 11-6. Planilha para inserção dos dados sobre o caramujo Littoraria angulifera.

\begin{tabular}{|c|c|c|c|c|c|c|c|}
\hline \multicolumn{8}{|c|}{ Littoraria angulifera } \\
\hline $\begin{array}{l}\text { Estação: } \\
\text { Ano: } \\
\text { Campanha: }\end{array}$ & & & & & & & \\
\hline \multicolumn{8}{|c|}{ Sítio 1} \\
\hline Transecto & Indivíduo & LC & $\mathrm{AC}$ & $\mathrm{PT}$ & PU & PS & PSLC \\
\hline \multicolumn{8}{|l|}{ A } \\
\hline & & & & & & & \\
\hline \multicolumn{8}{|c|}{ Sítio 2} \\
\hline Transecto & Indivíduo & LC & $\mathrm{AC}$ & $\mathrm{PT}$ & PU & PS & PSLC \\
\hline B & & & & & & & \\
\hline & & & & & & & \\
\hline \multicolumn{8}{|c|}{ Sítio 3} \\
\hline Transecto & Indivíduo & LC & $\mathrm{AC}$ & $\mathrm{PT}$ & $\mathrm{PU}$ & PS & PSLC \\
\hline & & & & & & & \\
\hline \multicolumn{8}{|l|}{$\mathrm{C}$} \\
\hline & & & & & & & \\
\hline & & & & & & & \\
\hline $\begin{array}{l}\text { Legenda: LC } \\
\text { peso seco; P }\end{array}$ & argura d & , & TH & & & & ; PS = \\
\hline
\end{tabular}

\section{AUTORES}

Angelo F. Bernardino - Universidade Federal do Espírito Santo - UFES (autor para correspondência: angelo.bernardino@ufes.br)

Francisco Barros - Universidade Federal da Bahia - UFBA

José Souto R. Filho - Universidade Federal do Pernambuco - UFPE

Larisse F. Perez - Universidade Federal de Santa Catarina - UFSC

Leonir A. Colling - Universidade Federal do Rio Grande - FURG

Paulo C. Lana - Universidade Federal do Paraná - UFPR

Paulo R. Pagliosa - Universidade Federal de Santa Catarina - UFSC

Rafaela C. Maia - Instituto Federal de Educação, Ciência e Tecnologia do Ceará IFCE

Ronaldo A. Christofoletti - Universidade Federal de São Paulo - UNIFESP

Sergio A. Netto - Universidade do Sul de Santa Catarina - UNISUL

Tânia M. Costa - Universidade Estadual Paulista - UNESP 


\section{REFERÊNCIAS}

Angulo R.J.; Lessa G.C. 1997. "The Brazilian sea level curves: a critical review with emphasis on the curves from Paranaguá and Cananéia regions”. Marine Geology, 140: 141-166.

Bernardino, A.F.; Pagliosa, P.R.; Christofoletti, R.A.; Barros, F.; Netto, S.A.; Muniz, P.; Lana, P.C. 2015. "Benthic estuarine communities in Brazil: moving forward to long term studies to assess climate change impacts". Brazilian Journal of Oceanography.

Bernardino, A.F.; Netto, S.A.; Pagliosa, P.R.; Barros, F.; Christofoletti, R.A.; Rosa Filho, J.S.; Colling, A.; Lana, P.C., no prelo. "Predicting ecological changes on benthic estuarine assemblages through decadal climate trends along Brazilian Marine Ecoregions". Estuarine Coastal and Shelf Science.

Brey, T. Population dynamics in benthic invertebrates. A virtual handbook. Alfred Wegener Institute for polar and marine research, Germany. Version 01.2, 2001, disponível em <http://www.thomas-brey.de/science/ virtualhandbook>

Gilman, E.L.; Ellison, J.; Duke, N.C.; Field, C. 2008. "Threats to mangroves from climate change and adaptation options: A review". Aquatic Botany, 89: 237-250.

IPCC, 2007: "Summary for Policymakers". In: Climate Change 2007: The Physical Science Basis. Contribution of Working Group I to the Fourth Assessment Report of the Intergovernmental Panel on Climate Change [Solomon, S., D. Qin, M. Manning, Z. Chen, M. Marquis, K.B. Averyt, M.Tignor and H.L. Miller (eds.)]. United Kingdom and New York, NY, USA: Cambridge University Press, Cambridge.

Marengo, J.A.; Ambrizzi, T.; Rocha, R.P.; Alves, L.M.; Cuadra, S.V.; Valverde, M.C.; Torres, R.R.; Santos, D.C.; Ferraz, S.E.T. 2010. "Future change of climate in South America in the late twenty-first century: inter comparison of scenarios from the three regional climate models". Climate Dynamics, 35: 10731097.

McGlone, D.; Vuille, M. 2012. "The associations between El Niō-Southern Oscillation and tropical South American climate in a regional climate model”. Journal of Geophysical Research, 117, D06105, doi:10.1029/2011JD017066.

McLusky, D.S.; Eliott, M. 2006. The estuarine ecosystem, ecology, threats and management. $3^{\mathrm{a}}$ Ed. Oxford: Oxford University Press, p. 208.

Meier, M.F.; Dyurgerov, M.B.; Rick, U.K.; O’Neel, S.; Pfeffer, W.T.; Anderson, R.S.; Anderson. S.P.; Glazovsky, A.F. 2007. "Glaciers dominate eustatic sea-level rise in the 21st century". Science, 317: 1064.

Melo, G.A.S. 1996. Manual de identificação dos Brachyura (caranguejos e siris) do litoral brasileiro. São Paulo: Plêiade-FAPESP, p. 603.

Nicholls, R.J.; Hoozemans, F.M.J.; Marchand, M. 1999. "Increasing flood risk and wetland losses due to global sea-level rise: regional and global analyses". Global Environmental Change, 9: S69-S87.

Raicich, F. (2008). "A review of sea level observations and low frequency sea-level variability in South Atlantic". Physics and Chemistry of the Earth, 33: 239-249.

Schaeffer-Novelli, Y.; Cintrón-Molero, G.; Adaime, R. R.. 1990. "Variability of mangrove ecosystems along the Brazilian coast". Estuaries, 13(2): 204-218.

Thurman, C.L.; Faria, S.C.; McNamara, J. 2013. "The distribution of fiddler crabs $(U c a)$ along the coast of Brazil: implications for biogeography of the western Atlantic Ocean”. Marine Biodiversity Records, 6: 1-21. 


\section{ANEXo 11-I - GLOSSÁRIO}

\section{ANEXOS - CAPÍTULO 11}

\section{BORDA}

Área localizada na divisa entre dois habitats.

\section{ESTUÁRIO}

Ecossistema de interface entre continentes-oceano caracterizado pela mistura de água doce de rios e riachos com água salgada do oceano (Fairbridge, 1980; McLusky \& Eliott, 2006).

\section{HABITAT}

Porção de um ambiente com características estruturais visualmente diferenciadas. Por exemplo, no protocolo de Estuários, categorizados como "vegetados" ou "não-vegetados".

\section{REGIÃO}

Escala espacial mais ampla utilizada neste protocolo separando as regióes Norte, Nordeste, Sudeste e Sul do Brasil.

\section{SETOR}

Divisão do estuário de acordo com sua amplitude salina média (em PSU), sendo dividida em baixo estuário (salinidade média de 20), médio estuário (salinidade média próxima a 10) e alto estuário (salinidade média abaixo de 10).

\section{Sítio}

Local de coleta (Exemplo, Praia Cabelo Gordo, Estuário da Baía de Paranaguá etc.).

\section{REFERÊNCIAS}

Fairbridge, R. 1980. "The estuary: its definition and geodynamic cycle”. In E. Olausson and I. Cato (Eds.), Chemistry and Geochemistry of Estuaries, 1-35. New York: John Wiley and Son.

McLusky, D.S.; Eliott, M. 2006. The estuarine ecosystem, ecology, threats and management. $3^{\text {a }}$ Ed. Oxford: Oxford University Press, p. 208. 


\section{MANGUEZAIS}

\section{OBjeTivos}

Os métodos para estudos e acompanhamento da dinâmica de bosques já são mundialmente consolidados (Cintrón \& Schaeffer-Novelli, 1984; Maia \& Coutinho, 2012) e serão utilizados neste protocolo apenas para caracterizar as áreas de estudo do GT Estuários. Os acompanhamentos da dinâmica de bosques frente a efeitos de mudanças climáticas será realizado pelo GT Manguezais e Marismas (Capítulo 4).

\section{MÉTodos}

Em cada sítio de trabalho sáo delineadas parcelas de 10x10 m, distribuídas aleatoriamente em um único gradiente de inundação a 10 metros de distância da margem do bosque. Em cada parcela, todas as árvores de mangue sáo mensuradas utilizando-se as seguintes medidas:

- Circunferência à Altura do Peito (CAP): a circunferência do fuste tomada à altura do peito do observador, mais especificamente a 1,3 m do solo. No caso dos indivíduos de Rhizophora que possuírem raízes escoras acima de 1,30 m, o CAP é mensurado acima da última raiz escora;

- Altura Total (AT): Compreende a distância entre a base da árvore e a extremidade da copa.

Das árvores mortas também são retidas a medida de CAP e todas as plântulas dentro das parcelas também são contadas.

Depois de concluída a etapa de campo, os dados servem de base para a estimativa dos seguintes parâmetros estruturais:

a) Diâmetro à Altura do Peito (DAP) - a partir dos valores da circunferência do fuste calcula-se o DAP para o gêneros Rhizophora, Avicennia e Laguncularia:

$$
D A P=\frac{C A P}{\pi}
$$

Para árvores que apresentam mais de um fuste, o DAP será estimado através da fórmula do Diâmetro Médio (DM):

$$
D M=\sqrt{\frac{(g)(12732,39)}{n}}
$$

Onde: $\mathrm{g}$ = somatório da área basal dos fustes

$\mathrm{n}=$ número de fustes

Os indivíduos registrados sáo subdivididos em classes de diâmetro e altura, com o objetivo de analisar o desenvolvimento das florestas estudadas. A quantidade e classe de diâmetro dos indivíduos mortos são úteis também para corroborar com a análise de desenvolvimento do bosque, bem como para averiguar o status de conservação deles.

b) Freqüência (F) - é o registro da presença de uma determinada espécie em uma dada parcela, de acordo com sua freqüência relativa (FR).

c) Densidade (De) - é o número de indivíduos por unidade de área (ind . ha-1), também representada como densidade relativa (DeR). 
d) Dominância (Do) - a dominância representa o reflexo da área basal de cada espécie em uma dada parcela. A área basal $\left(\mathrm{m}^{2}\right)$ para cada indivíduo é estimada através da seguinte fórmula:

$$
g=0,00007854 * D A P^{2}
$$

Esse parâmetro é também representado como dominância relativa (DoR) Relativa.

e) Valor de Importância - é a soma da Frequência Relativa, Densidade Relativa e Dominância

\section{MARISMAS}

Para efeito de padronização, apenas bancos de Spartina alterniflora serão amostrados. Métodos utilizados são similares aos descritos por Lana \& Guiss (1992).

Ao longo do litoral brasileiro as marismas variam de poucos metros a dezenas de quilômetros de extensão (Figura 11-II-1). Considerando que, de acordo com o protocolo GT Estuários, cada sítio deve estar afastado a uma distância de pelo menos $50 \mathrm{~m}$ entre si, em alguns estados do Brasil bancos diferentes serão amostrados, ao passo que em outros, a amostragem ocorrerá dentro de um mesmo banco de $S$. alterniflora. Desse modo, duas descrições iniciais são importantes: (1) se a amostragem ocorreu em um único ou mais bancos; (2) qual o tamanho aproximado do(s) banco(s) amostrado(s). Uma estimativa de tamanho dentro de cada sítio pode ser feita com uma trena simples, quando o banco for pequeno, ou com uso de um GPS se muito extenso. Não se busca aqui precisóes milimétricas, mas sim uma medida aproximada de sua extensão com objetivo de comparação em grande escala (latitudinal).
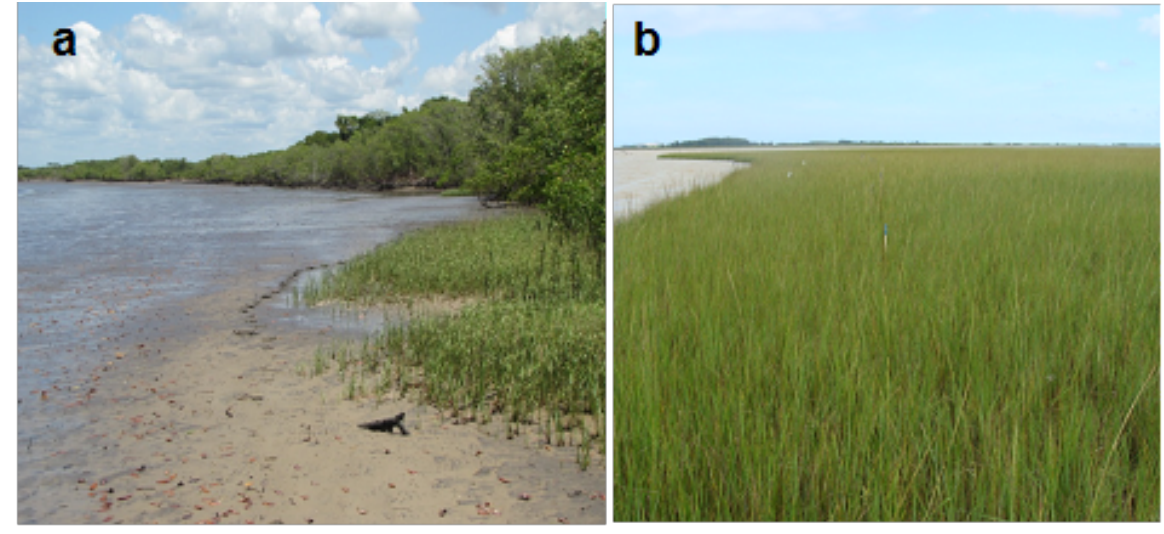

Figura 11-II-1. (a) Marismas de Spartina alterniflora como formaçóes pioneiras em faixas estreitas e descontínuas em franjas de manguezais (foto de formação em Coruripe, Ba) e (b) como formaçóes amplas e dominantes de faixas entremarés na ausência de manguezais (foto de formação em Laguna, SC).

Em cada sítio serão tomadas medidas de altura e de densidade das marismas dentro de uma área de $30 \mathrm{~cm}$ x $30 \mathrm{~cm}$. A medida de densidade representa o número total de colmos e folhas (Figura 11-II-2) dentro da área estabelecida. Já o registro da altura é dado pela distância do sedimento à porção mais alta da planta (Figura 11-II-2). Em cada sítio serão tomados dez registros de altura de S. alterniflora. 


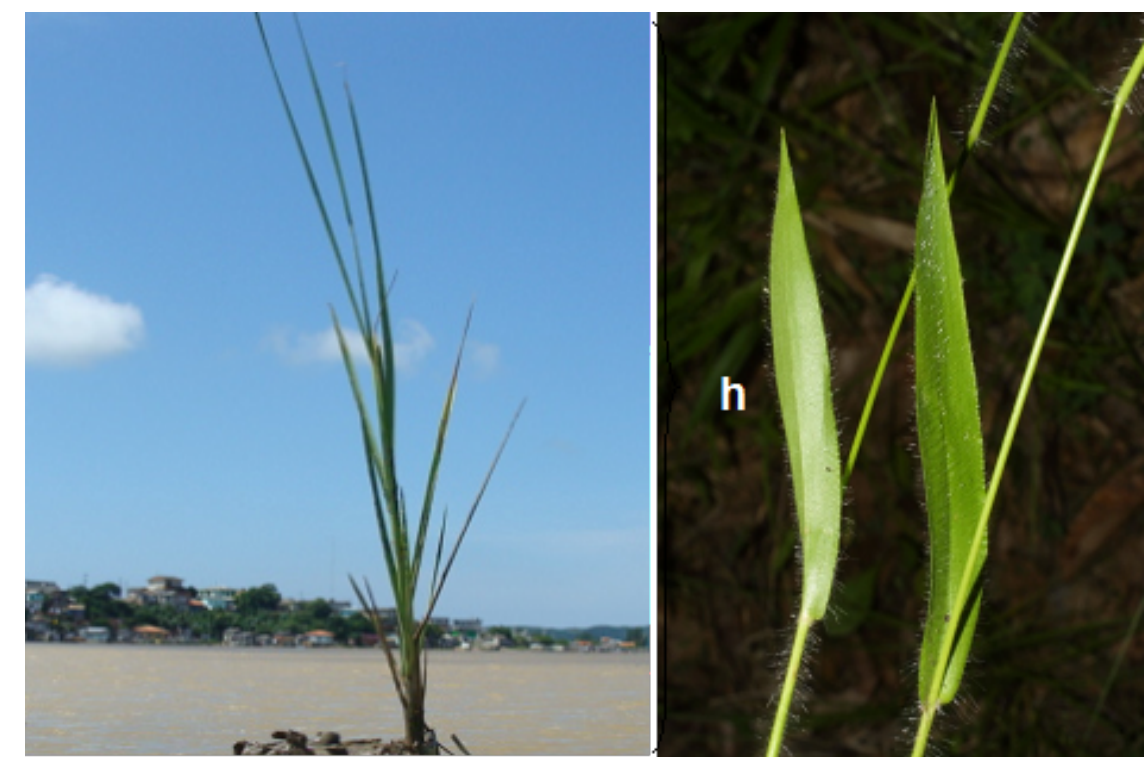

Figura 11-II-2. Colmos e folhas e medida de altura (h) de marismas.

\section{REFERÊNCIAS}

Cintrón, G.M.; Schaeffer-Novelli, Y. 1984. "Methods for studying mangrove structure”. In: Snedaker, S.C., Snedaker, J.G. (eds.). The mangrove Ecosystem: research methods. Monographs in Oceanographic Methodology, 8: 91-113.

Lana, P. C.; Guiss, C. 1992. "Macrofauna - plant-biomass interactions in a euhaline salt marsh in Paranagua Bay (SE Brazil)". Marine Ecology Progress Series, 80: 54-64.

Maia, R.C.; Coutinho, R. 2012. "Structural characteristics of mangrove forests in Brazilian estuaries: a comparative study". Revista de Biologia Marina y Oceanografia, 47 (4): 87-98. 


\section{Anexo 11-III. Protocolo para Estimativa da Produção Secundária MACROBENTônica}

\section{OBjetivos}

Propor um protocolo para estimar a produção secundária da comunidade bentônica ao longo da costa do Brasil.

\section{MATERIAL E EQUIPAMENTOS}

- Formol 4\%, peneiras de $500 \mu \mathrm{m}$, pinças, placa de petri, papel filtro, papel alumínio, potes de vidro com tampa, sacos plásticos, papel vegetal, pincel e lápis;

- Desumidificador, balança de precisão de 4 casas decimais, estufa e microscópio estereoscópico.

\section{MÉTodos}

O procedimento será realizado com todos os organismos (inteiros e pedaços), levando-se em consideração inclusive os organismos com conchas, como gastrópodes e bivalves.

- Após a coleta, fixar as amostras em formol 4\% e lavar o material sobre peneira;

- Em laboratório, separar o material biológico no nível taxonômico de Classe (segundo Brusca \& Brusca, 2007). Adicionalmente, separar os poliquetas em Scolecida, Canalipalpata e Aciculata (Rouse \& Pleijel, 2008); os crustáceos em Caridea, Stenopodidae, Brachyura, Anomura, Thalassinidae, Cumacea, Tanaidacea, Isopoda, Amphipoda e Ostracoda e os moluscos em bivalves e gastrópodes (Brusca \& Brusca, 2007).

\section{Estimativa da Biomassa}

Durante o primeiro ano, com amostragens mais detalhadas temporalmente, serão estimadas as biomassas em peso úmido, peso seco e peso seco livre de cinzas a fim de avaliar a variabilidade entre as medidas nos diferentes locais da costa e estabelecer um fator de conversão entre essas medidas.

\section{Peso Úmido (PU)}

- Identificar e pesar (P1) uma caixinha feita de papel alumínio para cada grupo animal de cada amostra (grupo/amostra). Se necessário, manter a amostra no desumidificador;

- Secar rapidamente em papel filtro cada conjunto de indivíduos do grupo/amostra, a fim de retirar o excesso de água;

- Colocar cada grupo/amostra previamente seco na sua caixinha e pesá-lo (P2).

$$
P U=P 2-P 1
$$

\section{Peso Seco (PS)}

- Colocar cada grupo/amostra na estufa a $60^{\circ} \mathrm{C}$ por $48 \mathrm{~h}$ ou até peso constante;

- Retirar cada grupo/amostra da estufa, mantendo-as no desumidificador até pesá-las (P3).

$$
P S=P U-(P 3-P 1)
$$




\section{Peso Seco Livre de Cinzas (PSLC)}

- Colocar cada grupo/amostra na mufla a $550^{\circ} \mathrm{C}$ por $4 \mathrm{~h}$;

- Retirar cada grupo/amostra da mufla, mantendo-as no desumidificador até pesá-las (P4).

$$
P S L C=P S-(P 4-P 1)
$$

\section{Planilha de Dados Brutos}

Organizar os dados para cada estimativa de biomassa em pastas separadas de uma planilha do Excel, com cada grupo/animal nas linhas (em ordem alfabética das Classes e táxons dentro das Classes) e cada amostra nas colunas.

\section{Cálculo da Produção Secundária}

- Baixar a planilha referente aos trabalhos de Brey (1999 e 2001) "Empirical Model: Production, P/B, mortality, and other parameters" - Version 4-04 (May '04) disponível em: <http://www.thomas-brey.de/science/virtualhandbook/spreadsheets/EmpRelat04.zip> Acesso em: 04 de maio de 2012;

- Todos os valores devem estar em kJ. Converter o valor multiplicando pelo fator de conversão que se encontra na pasta "Conversion" de acordo com o grupo analisado;

- Na pasta “BreyModelTab", inserir os valores médios de biomassa (Kj) e densidade (m2) nas colunas denominadas "Biomass $\mathrm{kJ} \mathrm{m}^{\wedge} 2$ " e "Population $\mathrm{N}$ ind $\mathrm{m}^{\wedge} 2$ ", respectivamente;

- Na coluna “Body Mass", dividir o valor de “Biomass $\mathrm{kJ} \mathrm{m}^{\wedge} 2$ " pelo de "Population $\mathrm{N}$ ind $\mathrm{m}^{\wedge} 2^{\prime \prime}$

- Depois, adicionar 0 ou 1 nas demais informaçóes (como temperatura, profundidade e etc.), conforme necessário;

- Criar nova pasta para juntar os dados de cada amostra e grupo/amostra.

\section{REFERÊNCIAS}

Brey, T., 1999. A collection of empirical relations for use in ecological modelling. NAGA The LARM Quarterly, 22(3): 24-28.

Brey, T. 2001. Population dynamics in benthic invertebrates. A virtual handbook. Version 01.2. Disponível em: $<$ http://www.thomas-brey.de/science/virtualhandbook>.

Brusca, R.C.; Brusca, G.J. 2007. Invertebrados. Rio de Janeiro: Guanabara. 2 Ed.

Rouse, G.W.; Pleijel, F. 2008. Polychaetes. Nova Iorque: Oxford. 


\section{OBJETIVO}

Fornecer uma metodologia para a análise do conteúdo de matéria orgânica em amostras de sedimentos estuarinos através da calcinação em forno mufla.

\section{Métodos}

- As amostras coletadas em campo devem ser armazenadas em sacos plásticos identificados e protegidas do sol (preferencialmente refrigeradas), até serem transportadas ao laboratório onde ficarão congeladas até a realização das análises;

- Descongelar, em temperatura ambiente, as amostras de sedimento nos sacos plásticos;

- Após descongelamento, homogeneizar cada amostra e colocar o sedimento em placa de petri pré-pesada e identificada. Pesar novamente e registrar (P1 = peso amostra úmida - peso placa);

- Secar amostras em estufa a temperatura de $60^{\circ} \mathrm{C}^{*(\mathrm{Obs} 1)}$, até alcançar peso constante ${ }^{1 .}$;

- Desagregar a amostra utilizando pistilo e almofariz ${ }^{1,2}$, durante período de secagem ${ }^{3}$;

- Resfriar amostra até alcançar temperatura ambiente em dessecador e pesar amostras secas (P2 = peso amostra seca - peso placa). É possível calcular o conteúdo de água pela diferença entre P1 e P2 final ${ }^{1}$;

- Após alcançar peso constante, transferir aproximadamente $5 \mathrm{~g}$ de sedimento seco para cadinho limpo, pré-pesado e identificado. Registrar o peso seco da amostra antes da calcinaçáo $\left(\mathrm{PS}_{\mathrm{pre}}\right)$, com maior precisão possível;

- Colocar cadinho+amostra no forno mufla e aquecer a temperatura entre 500 e $550^{\circ} \mathrm{C}$ por $4 \mathrm{~h}^{1,4^{* *}(\mathrm{Obs} 2)}$;

- Resfriar amostra em dessecador e pesá-la $a^{2}$ no dia seguinte. Registrar o peso seco da amostra após a calcinação $\left(\mathrm{PS}_{\mathrm{pos}}\right)$;

- Calcular o percentual de matéria orgânica (MO) perdida após calcinação utilizando-se a fórmula indicada por Heiri et al. (2001):

$$
\% M O_{\text {perdida }}=\frac{\left(P S_{\text {pré }}-P S_{\text {pós }}\right)}{P S_{\text {pré }}} * 100
$$

\footnotetext{
* (Obs1) Suguio (1973) apontou que sedimentos argilosos não devem ser aquecidos em temperaturas superiores a $60^{\circ} \mathrm{C}$ a fim de evitar o endurecimento do material e a modificação de argilo-minerais.

** (Obs2) Brower et al. (1997) e Heiri et al. (2001) indicaram que a temperatura deve ser entre 500 e $550^{\circ} \mathrm{C}$, sendo que a partir desta, outras reações além da queima da matéria orgânica podem ocorrer, prejudicando os dados. Boto \& Wellington (1984), Lara-Domínguez et al. (2005) e Maia (2010) analisaram sedimentos coletados em manguezais utilizando a temperatura de $450^{\circ} \mathrm{C}$. Tendo em vista essas informaçôes, foi definida a temperatura de $500^{\circ} \mathrm{C}$ para a calcinação de amostras de sedimento de manguezais no LEB.
} 


\section{REFERÊNCIAS}

(números sobrescritos ao longo da descrição do procedimento)

1 - Brower, J.E.; Zar, J.H.; von Ende, C.N. 1997. Field and laboratory methods for general ecology. 4a ed. WCB/ McGraw-Hill Companies, p. 273.

2-Dean, W.E. 1974. "Determination of carbonate and organic matter in calcareous sediments and sedimentary rocks by loss on ignition: comparison with other methods". Journal of Sedimentary Petrology, 44(1): 242-248.

3 - Sessegolo, G.C. 1997. Estrutura eprodução de serapilheira do manguezal do Rio Baguaçu, Baía de Paranaguá$P R$. Dissertação de Mestrado. Engenharia Florestal, Universidade Federal do Paraná. p. 10.

4-Heiri, O.; Lotter, A.F.; Lemcke, G. 2001. "Loss on ignition as a method for estimating organic and carbonate content in sediments: reproducibility and comparability of results". Journal of Paleolimnology, 25: 101-110.

5 - Suguio, K. 1973. Introdução à sedimentologia. Editora da Universidade de São Paulo. São Paulo: Edgar Blücher, p. 317.

6 - Boto, K.G.; Wellington, J.T. 1984. "Soil characteristics and nutrient status in a northern Australian mangrove forest". Estuaries, 7(1): 61-69.

7 - Lara-Domínguez, A.L.; Day Jr, J.W.; Zapata, G.V.; Twilley, R.R.; Guillén, H.A.; Yáñez-Arancibia, A. 2005. "Structure of a unique inland mangrove forest assemblage in fossil lagoons on the Caribbean Coast of Mexico". Wetlands Ecology and Management, 13: 111-122.

8 - Maia, R.C. 2010. O uso do gastrópode Melampus coffeus como indicador ecológico do estado de degradação ambiental dos manguezais do Ceará. Tese de Doutorado. Biologia Marinha, Universidade Federal Fluminense, p. 222. 